\title{
BOUNDARY LAYER FLOW WITH DIFFUSION AND FIRST-ORDER CHEMICAL REACTION OVER A POROUS FLAT PLATE SUBJECT TO SUCTION/INJECTION AND WITH VARIABLE WALL CONCENTRATION
}

\author{
Krishnendu Bhattacharyya* \\ Department of Mathematics, The University of Burdwan, Burdwan-713104, West Bengal, India
}

Received 18 November 2010; accepted 11 August 2011

\begin{abstract}
In this paper, an investigation is made to study the boundary layer flow over a porous flat plate with diffusion of chemically reactive species undergoing first-order reaction and subject to suction or injection. In this analysis, the variable plate concentration is considered. Using similarity variable technique, the governing partial differential equations are transformed into a set of self-similar ordinary differential equations which are solved by shooting method. The study reveals that for the increase of suction at the plate both velocity and concentration boundary layer thicknesses decrease and for increasing injection both thicknesses increase. The concentration at a fixed point within the boundary layer decreases with increasing values of the Schmidt number, the reaction rate parameter and the power-law exponent. For a fixed point, with increase of reaction rate parameter the magnitude of concentration gradient initially increases and then after a point it decreases. The rate of solute transfer from the plate increases with reaction rate parameter and power-law exponent.
\end{abstract}

Keywords: Boundary layer flow, diffusion, first-order chemical reaction, porous flat plate, suction/injection

DOI:10.3329/cerb.v15i1.6464

\section{Introduction}

The flow over a flat plate is a classical problem in fluid mechanics. A survey in the literature of boundary layer produces that the flow past a flat plate with uniform free stream is vastly studied. H. Blasius [1] first investigated the steady laminar boundary layer flow over a flat plate, using similarity variable he obtained the nonlinear third-order ordinary differential equation and finally solved it analytically. Few years later, Howarth [2] obtained the numerical solution of Blasius problem. Riley [3] discussed the flow of an electrically conducting fluid on a vertical plate in presence of strong magnetic field normal to the flow. The existence of a solution for flow past a flat plate was established by Abu-Sitta [4]. Watanabe and Pop [5] demonstrated the hall effect on MHD boundary layer flow over a continuous moving flat plate. The mixed convectional aspects of the flat plate flow were investigated by Afzal and Hussain [6] and Yao [7]. Some important characteristics of natural convection over a semi-infinite vertical plate in a porous medium was studied by Hsu and Cheng [8]. Mukhopadhyay and Layek [9] analyzed the radiation effects on forced convective flow and heat transfer over a porous plate in a porous medium. Wang [10] obtained an approximate

\footnotetext{
*Corresponding author Email: krish.math@yahoo.com; krishnendu84@yahoo.com
}

solution of the classical Blasius equation using Adomian decomposition method (ADM). A numerical investigation of the classical Blasius flat plate problem was presented by Cortell [11]. Batallar [12] extended the Blasius problem by studying the effects of radiation on the boundary layer. Recently, Chowdhury [13] discussed the unsteady free convective flow of an electrically conducting viscoelastic fluid over an infinite non-conducting vertical plate in presence of transverse magnetic field.

The mass transfer analysis in boundary layer flow bears a great importance in extending the theory of separation processes and chemical kinetics. The diffusion of a chemically reactive species in a laminar boundary layer flow over a flat plate was discussed by Chambre and Young [14]. After that many researcher investigated the heat and mass transfer effects on the flow dynamics with and without chemical reaction. Gebhart and Pera [15] analysed the combined buoyancy effects of thermal and mass diffusion on vertical natural convection. The mass transfer effect on the flow past an impulsively started infinite vertical plate under several conditions were studied by Soundalgekar [16], Soundalgekar et al. [17], Das et al. [18] and Muthucumaraswamy and Ganesan [19, 20]. Andersson et al. [21] explained the diffusion of a chemically reactive species from a stretching sheet. Fan et al. [22] obtained the similarity solution of diffusion of chemically reactive species in mixed convection flow 
over a horizontal moving plate. Anjalidevi and Kandasamy $[23,24]$ studied the effects of chemical reaction, heat and mass transfer on laminar flow along a semi infinite horizontal plate and also analyzed the effects of a chemical reaction on the flow past a semi infinite plate in presence of a magnetic field. Recently, Postelnicu [25] described the influence of chemical reaction on heat and mass transfer by natural convection from vertical surface in porous media by taking into account the Soret and Dufour effects.

In the present paper, the effect of diffusion of chemically reactive species undergoing first-order chemical reaction on laminar boundary layer flow over a porous flat plate subject to suction or injection is investigated. In the analysis, the wall concentration is variable and the reaction rate is taken inversely proportional to the position along the plate. By using similarity transformation, self-similar set of equations are obtained and then solved numerically using well-known shooting method for the solution of boundary value problem (BVP). The numerical results are plotted and the variation of flow behaviour and solute transfer characteristics are thoroughly analysed.

\section{Formulation of the Problem}

Consider the steady two-dimensional flow of a viscous incompressible fluid and mass diffusion with first-order chemical reaction over a porous flat plate with suction or injection. Using boundary layer approximation, the equations for the flow and the concentration distribution may be written in usual notation as

$$
\begin{gathered}
\frac{\partial u}{\partial x}+\frac{\partial v}{\partial y}=0 \\
u \frac{\partial u}{\partial x}+v \frac{\partial u}{\partial y}=v \frac{\partial^{2} u}{\partial y^{2}} \\
u \frac{\partial C}{\partial x}+v \frac{\partial C}{\partial y}=D \frac{\partial^{2} C}{\partial y^{2}}-R\left(C-C_{\infty}\right)
\end{gathered}
$$

where $u$ and $v$ are velocity components in $x$ - and $y$-directions respectively, $v(=\mu / \rho)$ is the kinematic viscosity of fluid, $\rho$ is the density of fluid, $\mu$ is the coefficient of fluid viscosity, $C$ is the concentration, $D$ is the diffusion coefficient, $C_{\infty}$ is concentration in the free stream. $R(x)$ is the variable reaction rate of the solute and is given by $R(x)=L R_{0} / x, L$ is the reference length and $R_{0}$ is a constant.

The appropriate boundary conditions for the velocity components and the concentration are given by:

$$
\begin{gathered}
u=0, v=v_{w} \text { at } y=0 ; u \rightarrow U_{\infty} \text { as } y \rightarrow \infty \\
C=C_{w}=C_{\infty}+C_{0} x^{n} \text { at } y=0 ; C \rightarrow C_{\infty} \text { as } y \rightarrow \infty
\end{gathered}
$$

where $U_{\infty}$ is the free stream velocity, $C_{w}$ is the variable plate concentration with $C_{0}>0$ is a constant, $n$ is a power-law exponent signifies the change of amount of solute in the $x$-direction and $v_{w}$ is variable suction or injection through the porous plate and is given by $v_{w}=$ $v_{0} /(x)^{1 / 2}, v_{0}$ is a constant with $v_{0}<0$ for suction and $v_{0}>0$ for injection.

The stream function $\psi(x, y)$ can be introduced as:

$$
u=\frac{\partial \psi}{\partial y} \text { and } v=-\frac{\partial \psi}{\partial x}
$$

Using relation (6), the equation (1) is satisfied identically and the equations (2) and (3) become:

$$
\begin{gathered}
\frac{\partial \psi}{\partial y} \frac{\partial^{2} \psi}{\partial x \partial y}-\frac{\partial \psi}{\partial x} \frac{\partial^{2} \psi}{\partial y^{2}}=v \frac{\partial^{3} \psi}{\partial y^{3}} \\
\frac{\partial \psi}{\partial y} \frac{\partial C}{\partial x}-\frac{\partial \psi}{\partial x} \frac{\partial C}{\partial y}=D \frac{\partial^{2} C}{\partial y^{2}}-\frac{L R_{0}}{x}\left(C-C_{\infty}\right)
\end{gathered}
$$

The boundary conditions in (4) for the flow reduce to:

$$
\frac{\partial \psi}{\partial y}=0, \frac{\partial \psi}{\partial x}=-v_{w} \text { at } y=0 ; \frac{\partial \psi}{\partial y} \rightarrow U_{\infty} \text { as } y \rightarrow \infty
$$

The dimensionless variables for $\psi$ and $C$ can be introduced:

$$
\psi=\sqrt{U_{\infty} v x} f(\eta) \text { and } C=C_{\infty}+\left(C_{w}-C_{\infty}\right) \phi(\eta)
$$

where the similarity variable $\eta$ is defined as $\eta=$ $y\left(U_{\infty} / v x\right)^{1 / 2}$.

In view of relations in (10) we finally obtain the selfsimilar equations in the following form:

$$
\begin{gathered}
f^{\prime \prime \prime}+\frac{1}{2} f f^{\prime \prime}=0 \\
\phi+\frac{1}{2} \operatorname{Sc} f \phi^{\prime}-\operatorname{Sc}\left(n f^{\prime}+\beta\right) \phi=0
\end{gathered}
$$

where $\mathrm{Sc}=v / D$ is the Schmidt number and $\beta=$ $L R_{0} / U_{\infty}$ is the reaction rate parameter.

The boundary conditions (9) and (5) finally become:

$$
\begin{gathered}
f(\eta)=S, f^{\prime}(\eta)=0 \text { at } \eta=0 ; f^{\prime}(\eta) \rightarrow 1 \text { as } \eta \rightarrow \infty \\
\phi(\eta)=1 \text { at } \eta=0 ; \phi(\eta) \rightarrow 0 \text { as } \eta \rightarrow \infty
\end{gathered}
$$

where $S=\left(-2 v_{w} / U_{\infty}\right)\left(R e_{x}\right)^{1 / 2}=-2 v_{0} /\left(U_{\infty} v\right)^{1 / 2}$ is the suction or injection parameter. $S>0$ (i.e. $v_{0}<0$ ) corresponds to suction and $S<0$ (i.e. $v_{0}>0$ ) corresponds to injection.

\section{Numerical Method for Solution}

The nonlinear coupled differential equations (11) and (12) along with the boundary conditions form a BVP and is solved using shooting method, by converting it into an initial value problem (IVP). In this 


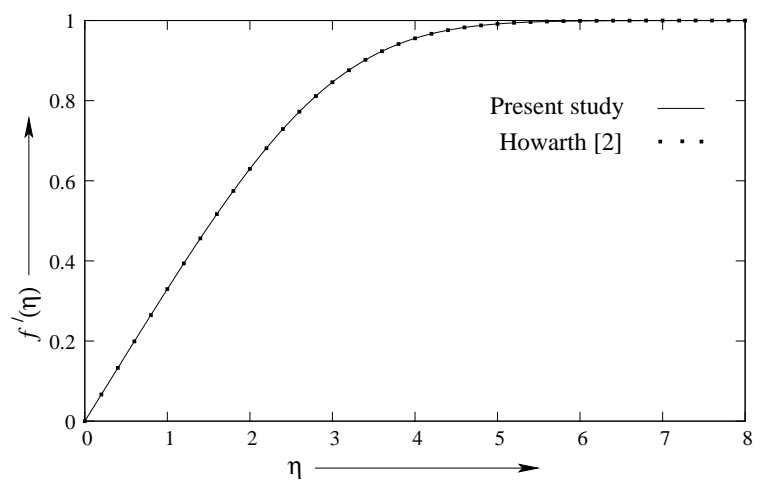

Figure 1: Velocity profile $f^{\prime \prime}(\eta)$ for $S=0$

method we have to choose a suitable finite value of $\eta \rightarrow \infty$, say $\eta_{\infty}$. We set following first-order system:

$$
\begin{gathered}
f^{\prime}=p, p^{\prime}=q, q^{\prime}=-\frac{1}{2} f q \\
\phi^{\prime}=z, z^{\prime}=-\frac{1}{2} \operatorname{Sc} f z+\operatorname{Sc}(n p+\beta) \phi
\end{gathered}
$$

with the boundary conditions:

$$
f(0)=S ; p(0)=0 ; \phi(0)=1
$$

To solve (15) and (16) with (17) as an IVP we must need values for $q(0)$ i.e. $f^{\prime \prime}(0)$ and $z(0)$ i.e. $\phi^{\prime}(0)$ but no such values are given in the boundary conditions. The initial guess values for $f^{\prime \prime}(0)$ and $\phi^{\prime}(0)$ are chosen and applying fourth order Runge-Kutta method a solution is obtained. Then we compare the calculated values of $f^{\prime}(\eta)$ and $\phi(\eta)$ at $\eta_{\infty}(=20)$ with the given boundary conditions $f^{\prime}\left(\eta_{\infty}\right)=1$ and $\phi\left(\eta_{\infty}\right)=0$ and adjust the values of $f^{\prime \prime}(0)$ and $\phi^{\prime}(0)$ using Secant method to give better approximation for the solution. The step-size is taken as $\Delta \eta=0.01$. The process is repeated until we get the results correct up to the desired accuracy of $10^{-6}$ level.

\section{Results and Discussion}

The numerical computations is carried out for several values of parameters involved in the equations viz. the suction or injection parameter $(S)$, the Schmidt number $(\mathrm{Sc})$, the reaction rate parameter $(\beta)$ and the power-law exponent $(n)$. The computed results are explained by plotting some figures and corresponding physical reasoning are also given.

At first, for the confirmation of the accuracy of applied numerical method we compare our obtained results corresponding to the velocity profile for $S=0$ (i.e. in absence of suction or injection) with the given result of Howarth [2] in Figure 1 and have found in excellent agreement.

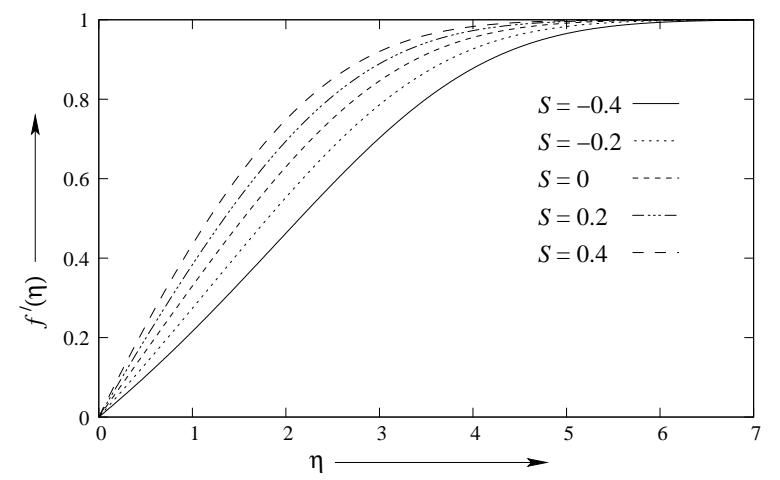

Figure 2: Velocity profiles $f^{\prime}(\eta)$ for various values of $S$

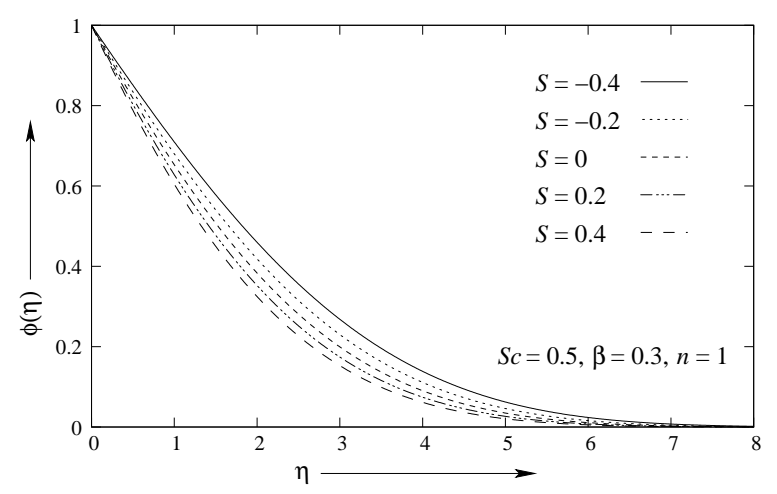

Figure 3: Concentration profiles $\phi(\eta)$ for various values of $S$

In the flow over a porous plate, the effects of externally applied suction or injection on velocity and concentration profiles are very important. The dimensionless velocity and concentration profiles are depicted in Figures 2 and 3 respectively for various values of the suction or injection parameter $S$. For the increase of applied suction, the value of the velocity profile $f^{\prime}(\eta)$ at a fixed $\eta$ increases and the concentration profile $\phi(\eta)$ decreases, it is due to the fact that the momentum as well as concentration boundary layer thicknesses decrease with suction. On the other hand, reverse scenario is observed for external injection case, that is, with increasing injection, the value of the velocity decreases and the concentration increases because due to the injection both boundary layer thicknesses increase.

We now concentrate our attention on the effect of the Schmidt number Sc on the reactive solute distribution. Figure 4 is the graphical representation of the concentration profiles for various values of Sc. The value of the solute profile at a fixed $\eta$ quickly decreases with increasing values of Sc. Actually the Schmidt number is inversely proportional to the diffusion coefficient. So, an increment of Sc produces a reduction of diffusion coefficient and which acts in thinning the concentration boundary layer thickness. For this reason the concentration profile decreases. Furthermore, an interesting characteristic of the concentration pro- 


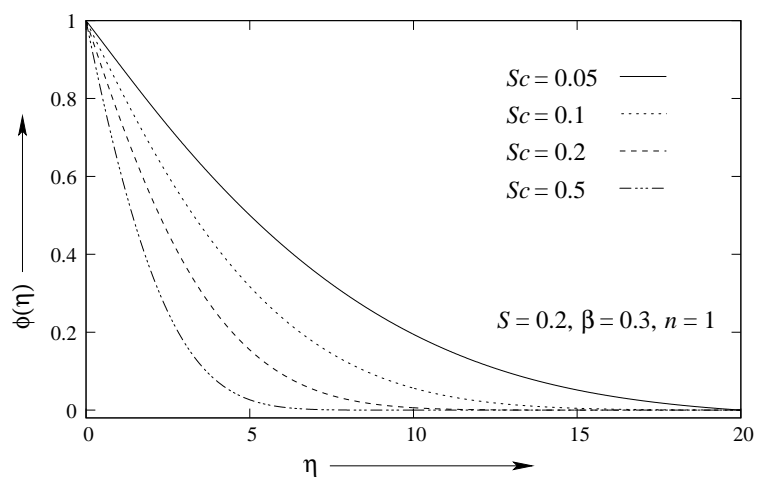

Figure 4: Concentration profiles $\phi(\eta)$ for various values of Sc

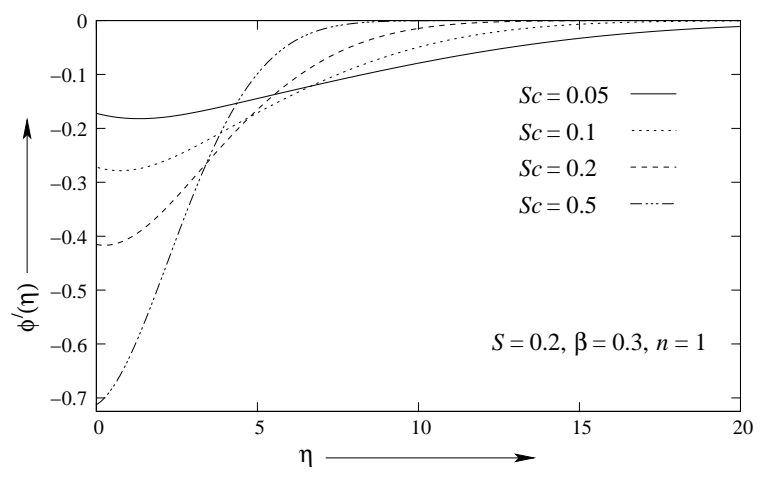

Figure 5: Concentration gradient profiles $\phi^{\prime}(\eta)$ for various values of $\mathrm{Sc}$

files is noticed i.e. after certain increment of Sc the solute profiles exhibit negative value $(\mathrm{Sc}=0.5)$ for some $\eta$. The negative value of the concentration profile for large Sc is because of substantial increase in the rate of solute transfer from the plate to the fluid in presence of chemical reaction. The concentration gradient profiles $\phi^{\prime}(\eta)$ for several values of Sc are plotted in Figure 5. It is observed from the figure that the magnitude of the concentration gradient initially increases with Sc, but for greater values of $\eta$ it decreases with Sc.

The effects of reaction rate parameter $\beta$ on the concentration profiles and concentration gradient profiles are presented by the Figures 6 and 7 . From the Figure 6 , we observed that the value of the solute profile at a fixed point decreases with increasing values of $\beta$. Thus the chemical reaction enhances the mass transfer. On the other hand, concentration gradient profiles exhibits different behaviour before and after a critical point. Before $\eta \approx 2.451$ the magnitude of concentration gradient profile increases and after that point it decreases. Furtherover, for small values of $\beta$ the magnitude of gradient curves at first increases for small $\eta$ and for greater values of $\eta$ it starts to decrease and finally approaches to zero. But, for large values of reaction rate parameter $\beta$, those curves decrease throughout and goes to zero.

In Figures 8 and 9, the variations of concentration

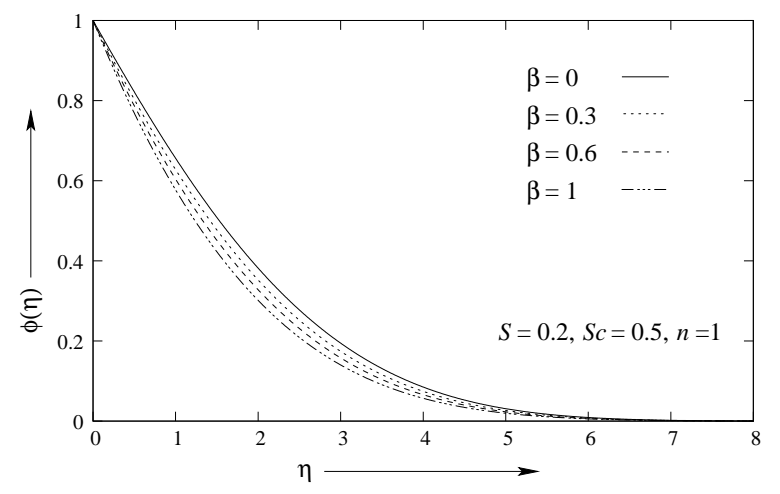

Figure 6: Concentration profiles $\phi(\eta)$ for various values of $\beta$

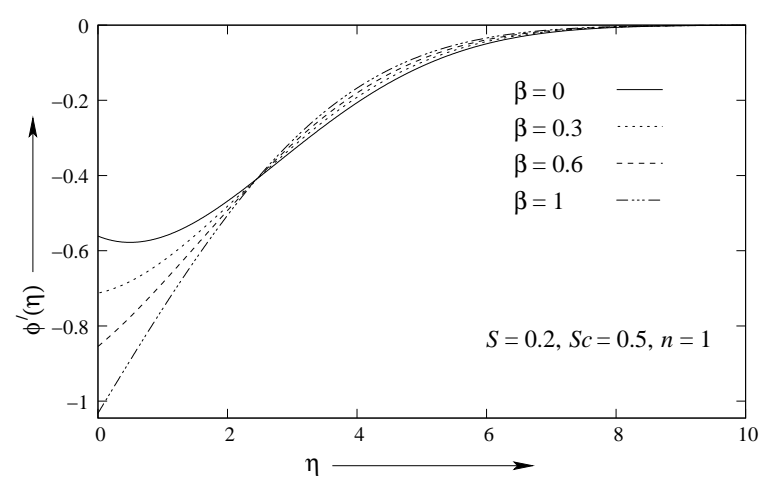

Figure 7: Concentration gradient profiles $\phi^{\prime}(\eta)$ for various values of $\beta$

and concentration gradient profiles due to the variation of power-law exponent $n$ are demonstrated. As similar to the reaction rate parameter, for increasing values of $n$ the concentration profile decreases. So, due to enhancement of plate concentration the solute transfer increases. The magnitude of the concentration gradient initially increases with increasing $n$, but for larger values of $\eta$ it decreases.

Finally, the values of $-\phi(0)$ which is proportional to the rate of mass transfer from the plate, for several values of reaction rate parameter $\beta$ and power-law exponent $n$ are depicted in Figure 10. It is seen that the

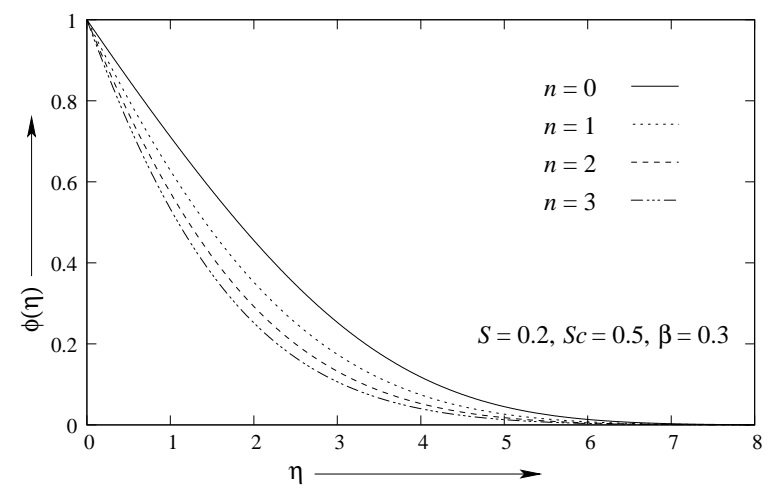

Figure 8: Concentration profiles $\phi(\eta)$ for various values of $n$ 


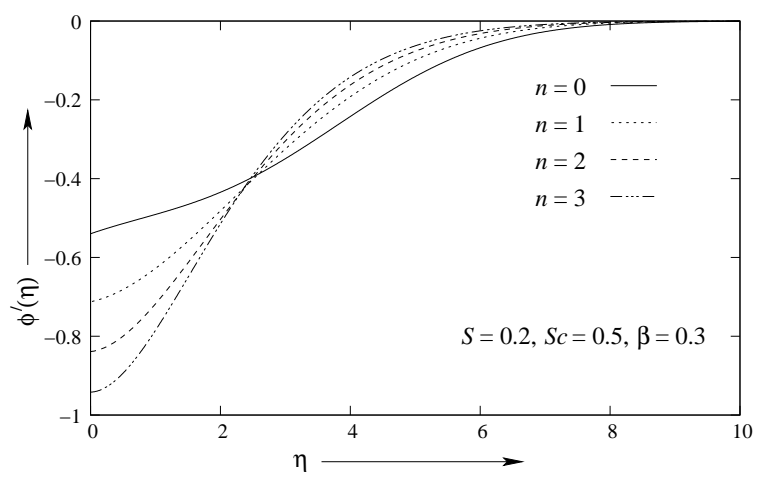

Figure 9: Concentration gradient profiles $\phi^{\prime}(\eta)$ for various values of

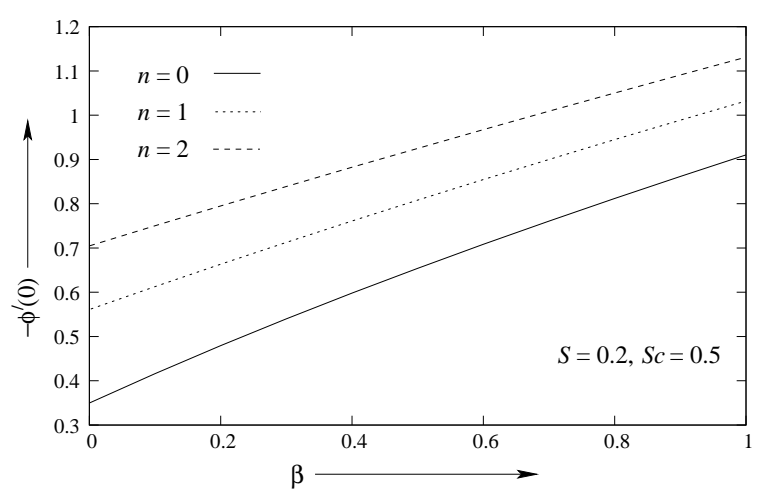

Figure 10: The values of $-\phi(0)$ against $\beta$ for various value of $n$

rate of mass transfer from the plate enhances for increments of both $\beta$ and $n$. It is very useful when the solute diffusion is given prime importance, i.e., in many processes in chemical engineering.

\section{Conclusion}

The objective of this investigation is to analyse the boundary layer flow over a porous flat plate with diffusion of chemically reactive species undergoing firstorder reaction and subject to suction/injection. In this analysis the plate concentration is taken variable. From the study these following points can be concluded.

- Due to increasing suction both velocity and concentration boundary layer thicknesses decrease, while for injection both increase.

- The increasing values of Schmidt number and the reaction rate parameter decreases the concentration at point.

- Most importantly, the increase of plate concentration enhances the rate of mass transfer from the plate.

\section{References}

[1] Blasius H, Grenzschichten in Flssigkeiten mit kleiner Reibung, Zeitschrift für Mathematik und Physik, 1908. 56:pp. 1-37

[2] Howarth L, On the solution of the laminar boundary layer equations, Proceedings of the Royal Society of London Series A, Mathematical and Physical Sciences, 1938. 164(919):pp. 547-579

[3] Riley N, Magnetohydrodynamic free convection, Journal of Fluid Mechanics, 1964. 18(4):pp. 577-586

[4] Abu-Sitta AMM, A note on a certain boundary-layer equation, Applied Mathematics and Computation, 1994. 64(1):pp. 73-77

[5] Watanabe T and Pop I, Hall effects on magnetohydrodynamic boundary layer flow over a continuous moving flat plate, Acta Mechanica, 1995. 108(1):pp. 35-47

[6] Afzal N and Hussain T, Mixed convection over a horizontal plate, Journal of Heat Transfer, 1984. 106(1):pp. 240-241

[7] Yao LS, Two-Dimensional Mixed Convection Along a Flat Plate, Journal of Heat Transfer, 1987. 109:pp. 440-445

[8] Hsu CT and Cheng P, The Brinkman model for natural convection about a semi-infinite vertical flat plate in a porous medium, International Journal of Heat and Mass Transfer, 1985. 28(3):pp. 683-697

[9] Mukhopadhyay S and Layek GC, Radiation effects on force convective flow and heat transfer over a porous plate in a porous medium, Mechanica, 2009. 44:pp. 587-597

[10] Wang L, A new algorithm for solving classical Blasius equation, Applied Mathematics and Computation, 2004. 157(1):pp. 1-9

[11] Cortell R, Numerical solutions of the classical Blasius flatplate problem, Applied Mathematics and Computation, 2005. 170(1):pp. 706-710

[12] Bataller RC, Radiation effects in the Blasius flow, Applied Mathematics and Computation, 2008. 198(1):pp. 333-338

[13] Chowdhury MMK, Effect of free convection flow of a viscoelastic fluid past an infinite vertical flat plate in presence of a transverse magnetic field, Chemical Engineering Research Bulletin, 2007. 10:pp. 11-31

[14] Chambre PL and Young JD, On the diffusion of a chemically reactive species in a laminar boundary layer flow, Physics of Fluids, 1958. 1:pp. 48-54

[15] Gebhart B and Pera L, The nature of vertical natural convection flows resulting from the combined buoyancy effects of thermal and mass diffusion, International Journal of Heat and Mass Transfer, 1971. 14(12):pp. 2025-2050

[16] Soundalgekar VM, Effects of Mass Transfer and FreeConvection Currents on the Flow Past an Impulsively Started Vertical Plate, Journal of Applied Mechanics, 1979. 46:pp. 757-760

[17] Soundalgekar VM, Birajdar NS and Darwhekar VK, Masstransfer effects on the flow past an impulsively started infinite vertical plate with variable temperature or constant heat flux, Astrophysics and Space Science, 1984. 100(1):pp. 159-164

[18] Das UN, Deka R and Soundalgekar VM, Effects of mass transfer on flow past an impulsively started infinite vertical plate with constant heat flux and chemical reaction, Forschung im Ingenieurwesen, 1994. 60(10):pp. 284-287

[19] Muthucumaraswamy R and Ganesan P, First-order chemical reaction on flow past an impulsively started vertical plate with uniform heat and mass flux, Acta Mechanica, 2001. 147(1):pp. $45-57$

[20] Muthucumaraswamy R and Ganesan P, On impulsive motion of a vertical plate with heat flux and diffusion of chemically reactive species, Forschung im Ingenieurwesen, 2000. 66(1):pp. $17-23$

[21] Andersson HI, Hansen OR and Holmedal B, Diffusion of a chemically reactive species from a stretching sheet, International Journal of Heat and Mass Transfer, 1994. 37(4):pp. 659-664

[22] Fan JR, Shi JM and Xu XZ, Similarity solution of mixed con- 
vection with diffusion and chemical reaction over a horizontal moving plate, Acta Mechanica, 1998. 126(1):pp. 59-69

[23] Anjalidevi SP and Kandasamy R, Effects of chemical reaction, heat and mass transfer on laminar flow along a semi infinite horizontal plate, Heat and Mass transfer, 1999. 35(6):pp. 465467

[24] Anjalidevi SP and Kandasamy R, Effects of chemical reaction, heat and mass transfer on MHD flow past a semi infinite plate, Zeitschrift für Angewandte Mathematik und Mechanik, 2000. 80(10):pp. 697-700

[25] Postelnicu A, Influence of chemical reaction on heat and mass transfer by natural convection from vertical surfaces in porous media considering Soret and Dufour effects, Heat and Mass Transfer, 2007. 43(6):pp. 595-602 